OPEN ACCESS

Edited by:

Giovanna Suzzi,

Università di Teramo, Italy

Reviewed by:

Odile Tresse

INRA - Centre Angers-Nantes, France

Giorgia Perpetuini,

Università di Teramo, Italy

*Correspondence:

Byeonghwa Jeon

bjeon@ualberta.ca

Specialty section:

This article was submitted to

Food Microbiology,

a section of the journal

Frontiers in Microbiology

Received: 15 January 2018

Accepted: 17 May 2018

Published: 06 June 2018

Citation:

Oh E, Andrews KJ and Jeon B (2018) Enhanced Biofilm Formation by Ferrous and Ferric Iron Through

Oxidative Stress in Campylobacter jejuni. Front. Microbiol. 9:1204. doi: 10.3389/fmicb.2018.01204

\section{Enhanced Biofilm Formation by Ferrous and Ferric Iron Through Oxidative Stress in Campylobacter jejuni}

\author{
Euna Oh, Katelyn J. Andrews and Byeonghwa Jeon* \\ School of Public Health, University of Alberta, Edmonton, AB, Canada
}

Campylobacter is a leading foodborne pathogen worldwide. Biofilm formation is an important survival mechanism that sustains the viability of Campylobacter under harsh stress conditions. Iron affects biofilm formation in some other bacteria; however, the effect of iron on biofilm formation has not been investigated in Campylobacter. In this study, we discovered that ferrous $\left(\mathrm{Fe}^{2+}\right)$ and ferric $\left(\mathrm{Fe}^{3+}\right)$ iron stimulated biofilm formation in Campylobacter jejuni. The sequestration of iron with an iron chelator prevented the iron-mediated biofilm stimulation. The level of total reactive oxygen species (ROS) in biofilms was increased by iron. However, the supplementation with an antioxidant prevented the total ROS level from being increased in biofilms by iron and also inhibited iron-mediated biofilm stimulation in C. jejuni. This suggests that iron promotes biofilm formation through oxidative stress. Based on the results of fluorescence microscopic analysis, $\mathrm{Fe}^{2+}$ and $\mathrm{Fe}^{3+}$ enhanced both microcolony formation and biofilm maturation. The levels of extracellular DNA and polysaccharides in biofilms were increased by iron supplementation. The effect of iron on biofilm formation was also investigated with $70 \mathrm{C}$. jejuni isolates from raw chicken. Regardless of the inherent levels of biofilm formation, iron stimulated biofilm formation in all tested strains; however, there were strain variations in iron concentrations affecting biofilm formation. The biofilm formation of $92.9 \%$ (65 of 70 ) strains was enhanced by either $40 \mu \mathrm{M} \mathrm{Fe}{ }^{2+}$ or $20 \mu \mathrm{M} \mathrm{Fe}^{3+}$ or both (the iron concentrations that enhanced biofilm formation in C. jejuni NCTC 11168), whereas different iron concentrations were required to promote biofilms in the rest of the strains. The findings in this study showed that $\mathrm{Fe}^{2+}$ and $\mathrm{Fe}^{3+}$ contributed to the stimulation of biofilm formation in $C$. jejuni through oxidative stress.

Keywords: Campylobacter, biofilms, oxidative stress, iron, survival mechanisms

\section{INTRODUCTION}

Campylobacter is a leading bacterial cause of gastroenteritis and is responsible for approximately 166 million diarrheal cases and 37,600 deaths worldwide per year (Kirk et al., 2015). In addition to gastrointestinal infections, in some cases, Campylobacter jejuni may result in the development of Guillain-Barré syndrome (GBS), an acute flaccid paralysis (Willison et al., 2016). Although C. jejuni is isolated from a wide range of domestic, companion, and wild 
animals (Huang et al., 2015), poultry is considered as the most important reservoir for foodborne transmission of $C$. jejuni to humans (Hermans et al., 2012). Compared to other foodborne pathogens, such as Salmonella and pathogenic Escherichia coli, C. jejuni is physiologically unique (e.g., microaerophilic and asaccharolytic) and fastidious to culture (Silva et al., 2011). Thus, specific culture conditions are required for the growth of C. jejuni. For example, low oxygen concentrations (e.g., 5\% $\mathrm{O}_{2}$ ) and high growth temperatures (e.g., $37 \sim 42^{\circ} \mathrm{C}$ ) are needed for the optimal growth of C. jejuni (Davis and DiRita, 2008). As a capnophile, additionally, $C$. jejuni requires $\mathrm{CO}_{2}$, and carbonic anhydrase that is encoded by $c a n B$ contributes to $C$. jejuni growth under low (such as 1\%) $\mathrm{CO}_{2}$ conditions (Al-Haideri et al., 2016).

A biofilm is microbial communities that are encased in a matrix of self-produced extracellular polymeric substance (EPS), including extracellular DNA (eDNA), polysaccharides, and proteins (Flemming et al., 2016). C. jejuni is capable of forming biofilms on various abiotic surfaces and frequently isolated from environmental samples (Kemp et al., 2005; Jokinen et al., 2011). Particularly, biofilm formation is deemed as an important survival mechanism in C. jejuni (Buswell et al., 1998; Murphy et al., 2006). As bacteria are usually found in biofilms in natural settings (Branda et al., 2005), Campylobacter is also found in biofilms on the surface of river rock and wood in the environment (Maal-Bared et al., 2012).

Several environmental factors affecting biofilm formation in C. jejuni have been reported. The biofilm formation of this microaerophilic bacterium is enhanced under aerobic conditions (Reuter et al., 2010; Turonova et al., 2015). Oxygen-rich conditions enhance the expression of membrane proteins, such as Peb4 and CadF, which are involved in the adhesion of C. jejuni to abiotic surfaces (Asakura et al., 2007; Sulaeman et al., 2012). Increased oxidative stress under aerobic conditions is associated with biofilm stimulation in C. jejuni (Oh et al., 2016). In addition, biofilm formation is also affected by nutritional factors. For instance, nutrient-rich culture media and high salt concentrations reduce biofilm formation in C. jejuni (Reeser et al., 2007). Iron is an essential nutrient required for all organisms (Chandrangsu et al., 2017) and is associated with biofilm formation in some bacteria, such as Streptococcus mutans (Berlutti et al., 2004). Staphylococcus aureus (Lin et al., 2012), and Pseudomonas aeruginosa (Banin et al., 2005). Since iron affects various biological processes in $C$. jejuni, such as gene expression regulation (e.g., Fur regulon) and protein glycosylation (e.g., $p g l A, p g l C$, and $p g l H$ ) (Palyada et al., 2004), we hypothesized that iron may be involved in biofilm formation in $C$. jejuni. To prove this hypothesis, in this study, we investigated the effect of ferrous $\left(\mathrm{Fe}^{2+}\right)$ and ferric $\left(\mathrm{Fe}^{3+}\right)$ iron on biofilm formation in C. jejuni NCTC 11168 and 70 C. jejuni strains isolated from raw chicken.

\section{RESULTS}

\section{Stimulation of Biofilm Formation by Iron}

To examine the effect of iron on biofilm formation, biofilm assays were performed with minimal essential medium alpha (MEM $\alpha$ ), which does not contain iron, with/without iron supplementation.

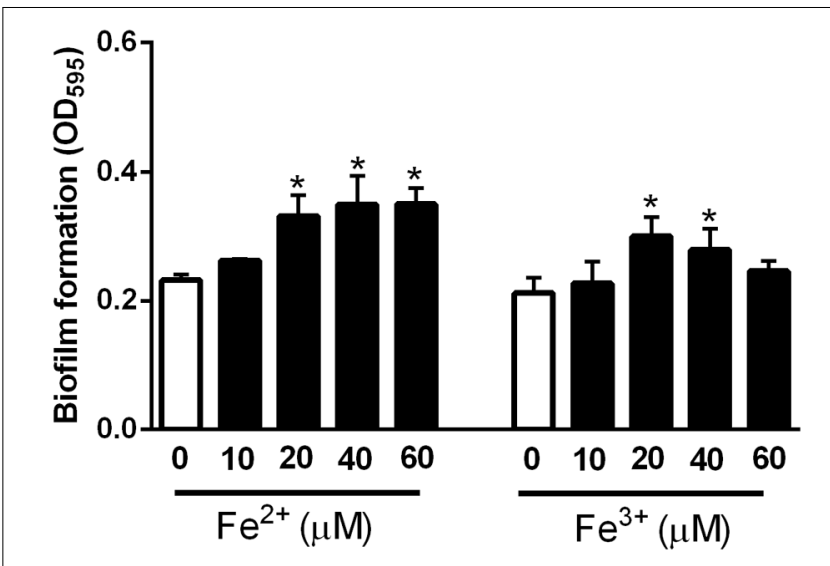

FIGURE 1 | Stimulation of biofilm formation by iron in C. jejuni NCTC 11168. The data show the means and standard deviations of three samples in a representative experiment. The experiments were repeated three times, and similar results were obtained in all repeated experiments. The statistical analysis was performed with Student's $t$-test in comparison with the non-treated sample. ${ }^{*} P<0.05$.

Interestingly, biofilm formation in C. jejuni was significantly enhanced by iron (Figure 1). Although both $\mathrm{Fe}^{2+}$ and $\mathrm{Fe}^{3+}$ affected biofilm formation in C. jejuni, $\mathrm{Fe}^{2+}$ and $\mathrm{Fe}^{3+}$ stimulated biofilm formation at different concentration ranges (Figure 1). Although the averages of bacterial counts in biofilms were slightly reduced at iron concentrations $\geq 20 \mu \mathrm{M}$, the reduction was not statistically significant, and the viability of C. jejuni in biofilms was not altered at the iron concentrations tested in the study (Supplementary Figure S1A). These results showed that iron, both $\mathrm{Fe}^{2+}$ and $\mathrm{Fe}^{3+}$, enhanced biofilm formation in C. jejuni.

\section{Inhibition of Iron-Mediated Biofilm Promotion by a Chelator and an Antioxidant}

To confirm the effect of iron on biofilm stimulation, biofilm assays were performed in the supplementation with an iron chelator. The treatment of biofilms with an iron chelator significantly inhibited the iron-mediated enhancement of biofilm formation in C. jejuni (Figure 2A). While iron is an essential nutrient, it may generate reactive oxygen species (ROS) through the Fenton/Haber-Weiss reaction (Cornelis et al., 2011). Since oxidative stress affects biofilm formation in C. jejuni (Oh and Jeon, 2014; Oh et al., 2016), we hypothesized that the iron-mediated biofilm promotion may be related to oxidative stress. To examine this hypothesis, we investigated the effect of antioxidant treatment on biofilm formation in the presence of iron. The levels of total ROS were increased by iron and reduced by an iron chelator and an antioxidant (Figure 2B). The intracellular levels of iron were increased by iron supplementation and reduced by an iron chelator (Figure 2C). Antioxidant treatment inhibited the iron-mediated promotion of biofilm formation, although the intracellular iron level of ironand antioxidant-treated biofilms was comparable to that in the biofilms treated with only iron (Figure 2C). Interestingly, biofilm 
formation was enhanced in proportion to the level of total ROS (Figures 2A,B), not that of intracellular iron (Figures 2A,C). The viability of $C$. jejuni in biofilms was not affected by the treatment conditions used in the study (Supplementary Figure S1B). These results suggested that biofilm promotion by iron is associated with oxidative stress in C. jejuni.

\section{Increased Production of EPS by Iron}

The formation of biofilms was observed in the presence and absence of iron using fluorescence microscopy. Iron supplementation significantly enhanced the establishment of microcolonies at the early stage $(12 \mathrm{~h})$ of biofilm formation and also increased the development of matured biofilm structures at $24 \mathrm{~h}$ (Figure 3), suggesting that iron may affect the early and late stages of biofilm formation in C. jejuni. To observe EPS production, biofilms were stained with $\mathrm{BOBO} 3$ and calcofluor white $(\mathrm{CW})$ to detect eDNA and extracellular polysaccharides, respectively. BOBO-3 is a DNAbinding red fluorescent dye and cannot penetrate through the membrane and thus is used to detect eDNA. CW is a fluorescent dye that binds to $\beta 1-3$ and $\beta 1-4$ carbohydrate linkages and has been used to detect polysaccharides in C. jejuni biofilms (McLennan et al., 2008). Both $\mathrm{Fe}^{2+}$ and $\mathrm{Fe}^{3+}$ substantially increased the production of eDNA and extracellular polysaccharides in biofilms; however, an iron chelator and an antioxidant reduced the levels of eDNA and extracellular polysaccharides (Figure 3). These findings demonstrated that iron promoted biofilm formation in C. jejuni by stimulating the production of eDNA and extracellular polysaccharides.

\section{Effect of Iron on Biofilm Formation in $\mathbf{7 0}$ Strains of C. jejuni From Retail Raw Chicken}

Using the iron concentrations determined with C. jejuni NCTC $11168\left(40 \mu \mathrm{M} \mathrm{Fe}{ }^{2+}\right.$ and $20 \mu \mathrm{M} \mathrm{Fe}^{3+}$; Figure 1), the effect of iron on biofilm formation was evaluated in $70 \mathrm{C}$. jejuni strains that were isolated from retail raw chicken in our previous study (Oh et al., 2015). The levels of biofilm formation in the tested strains varied significantly in the absence of iron, ranging from low (Figure 4A), medium (Figure 4B), to high levels (Figure 4C), and iron significantly stimulated biofilm formation in the tested strains with strain-dependent variations (Figure 4 and Supplementary Figure S2). Similar to C. jejuni NCTC 11168 (Figure 1), 51 (72.9\%) of the 70 tested strains exhibited biofilm promotion by both $40 \mu \mathrm{M} \mathrm{Fe}^{2+}$ and $20 \mu \mathrm{M} \mathrm{Fe}^{3+}$ (Figure 5A). However, biofilm formation in $14(20 \%)$ strains was enhanced by either only $40 \mu \mathrm{M} \mathrm{Fe} e^{2+}$ or $20 \mu \mathrm{M} \mathrm{Fe}{ }^{3+}$, not by both (Figure 5A), and biofilm formation in five strains $(7.1 \%)$ was promoted by neither $40 \mu \mathrm{M} \mathrm{Fe}^{2+}$ nor $20 \mu \mathrm{M} \mathrm{Fe}^{3+}$ (Figure 5A). The intrinsic level of biofilm formation was not correlated to the multilocus sequence typing (MLST) clonal complexes (CCs) of the strains. Overall, MLST CCs 21 and 45 were distributed in weak-, medium-, and strong-biofilm formers; however, minor MLST CCs, such as 353, 354, and 362, were not found in strongbiofilm formers (Figure 5B).
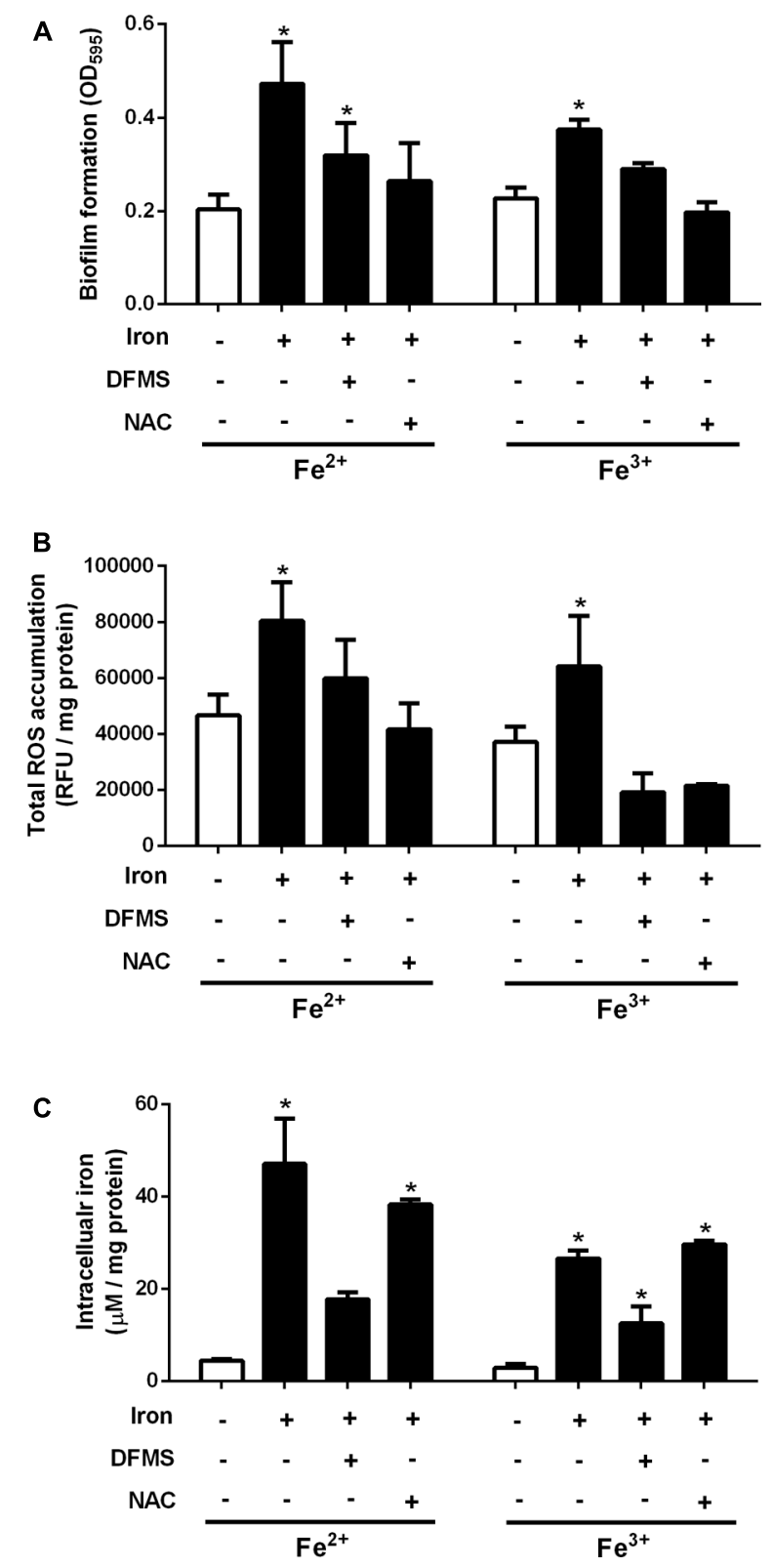

FIGURE 2 | Effects of iron, a chelator, and an antioxidant on the levels of biofilm formation (A), ROS production (B), and intracellular iron (C) in C. jejuni NCTC 11168. The concentrations of $\mathrm{Fe}^{2+}$ and $\mathrm{Fe}^{3+}$ were $40 \mu \mathrm{M}$ and $20 \mu \mathrm{M}$, respectively, which were determined based on the results of the biofilm assay (Figure 1). Twenty micromolar DFMS and $1 \mu \mathrm{M} \mathrm{N}$-acetylcysteine (NAC) were used as an iron chelator and an antioxidant, respectively. The results are the means and standard deviations of three samples in a representative experiment. The experiments were repeated three times, and similar results were observed in all repeated experiments. The statistical analysis was performed with Student's $t$-test compared to the non-treated sample. $* P<0.05$

Assuming strain variations in iron uptake and/or oxidative stress defense, we conducted biofilm assays at different iron concentrations with the 19 strains whose biofilm formation was not enhanced by either $40 \mu \mathrm{M} \mathrm{Fe}^{2+}$ or $20 \mu \mathrm{M} \mathrm{Fe}^{3+}$ or 


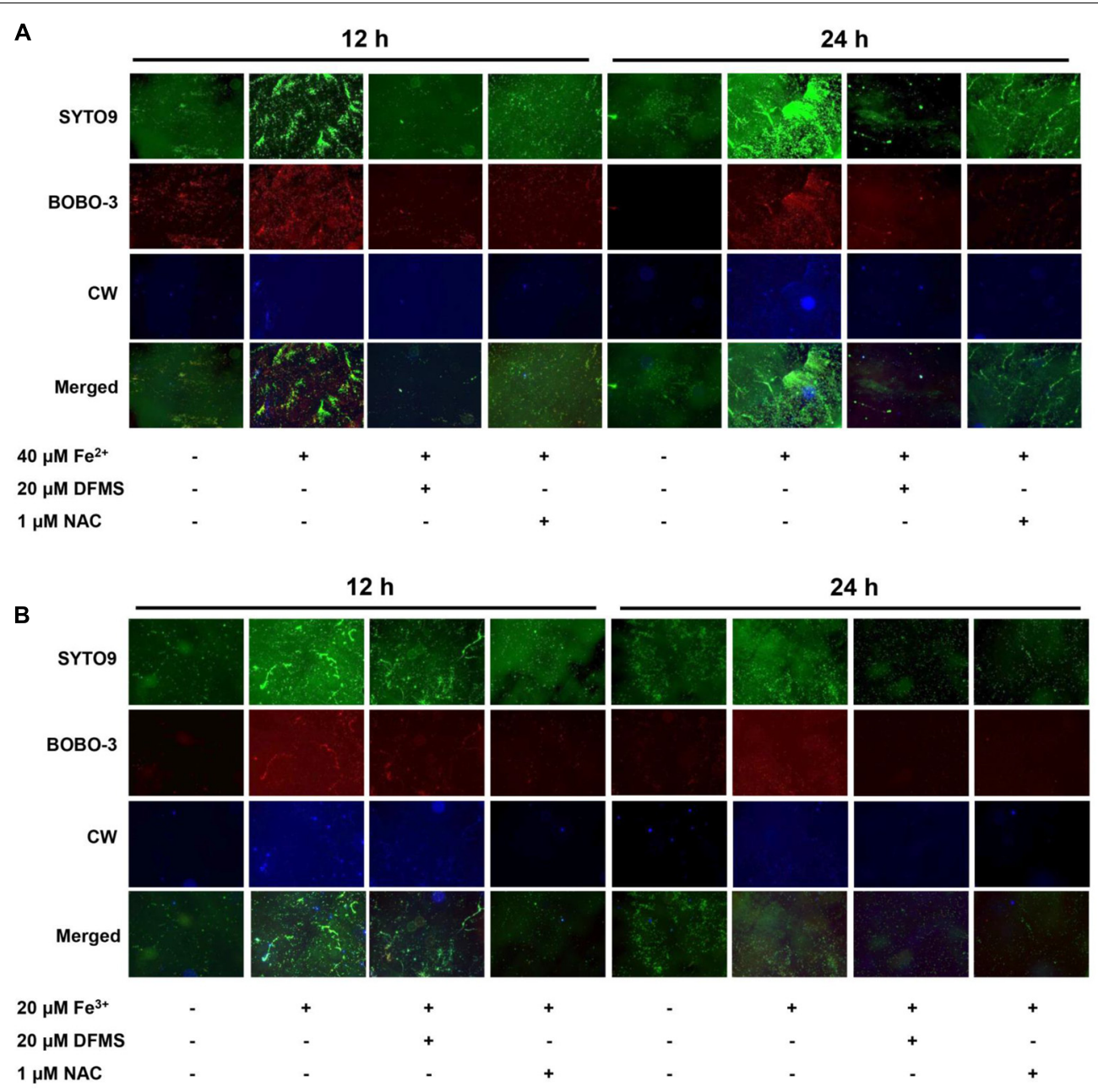

FIGURE 3 | Stimulatory effects of $\mathrm{Fe}^{2+}(\mathbf{A})$ and $\mathrm{Fe}^{3+} \mathbf{( B )}$ on biofilm formation in C. jejuni NCTC 11168. Biofilms were observed with fluorescence microscopy after staining with SYTO9, BOBO3, and calcofluor white (CW) to detect total DNA (i.e., biofilm structures), extracellular DNA, and extracellular polysaccharides, respectively. The experiments were repeated three times and generated similar results. DFMS and N-acetylcysteine (NAC) were used as an iron chelator and an antioxidant, respectively.

both (Figures 4, 5A). Interestingly, biofilm formation in all tested strains was enhanced by both $\mathrm{Fe}^{2+}$ and $\mathrm{Fe}^{3+}$ at different concentrations with substantial strain variations (Figure 6). These results show that most $C$. jejuni strains increased biofilm formation in similar concentration ranges (ca. $40 \mu \mathrm{M} \mathrm{Fe} \mathrm{Fe}^{2+}$ and $20 \mu \mathrm{M} \mathrm{Fe}^{3+}$ ). However, different iron concentrations were required for biofilm stimulation in some C. jejuni strains.

\section{DISCUSSION}

As an essential nutrient, iron is involved in various biological processes in C. jejuni (Palyada et al., 2004). $\mathrm{Fe}^{2+}$ may diffuse through outer-membrane porins and then pass through FeoB in the cytoplasmic membrane in C. jejuni (Naikare et al., 2006; Miller et al., 2009). $\mathrm{Fe}^{3+}$ uptake is mediated by multiple membrane transporters, whose expression is regulated by the ferric uptake regulator Fur (Miller et al., 2009). The findings in this study demonstrate that both $\mathrm{Fe}^{2+}$ and $\mathrm{Fe}^{3+}$ stimulate biofilm formation in C. jejuni. Effects of iron on biofilm formation have also been reported in other bacteria. Iron-depleted saliva increases aggregation and biofilm formation of S. mutans, an important pathogen causing dental caries (Berlutti et al., 2004). Iron enhances biofilm formation in S. aureus ATCC 35556 and a few clinical strains of $S$. aureus (Lin et al., 2012). However, the effect of iron on biofilm formation appears to be strain-dependent in $S$. aureus since iron reduces biofilm formation in $S$. aureus Newman (Johnson et al., 2005). Biofilm formation in most of the strains tested in this study was stimulated $40 \mu \mathrm{M} \mathrm{Fe}^{2+}$ and $20 \mu \mathrm{M}$ $\mathrm{Fe}^{3+}$ (Figures 4, 5A), which were effective at biofilm promotion in C. jejuni NCTC 11168 (Figure 1). In some other C. jejuni strains, $40 \mu \mathrm{M} \mathrm{Fe}^{2+}$ and $20 \mu \mathrm{M} \mathrm{Fe}^{3+}$ did not enhance biofilm formation, and different iron concentrations were required to promote biofilm formation in these strains (Figure 6). Variations in the iron concentrations impacting biofilm formation may be associated with the strain variations in iron-associated genes in 


\section{A}

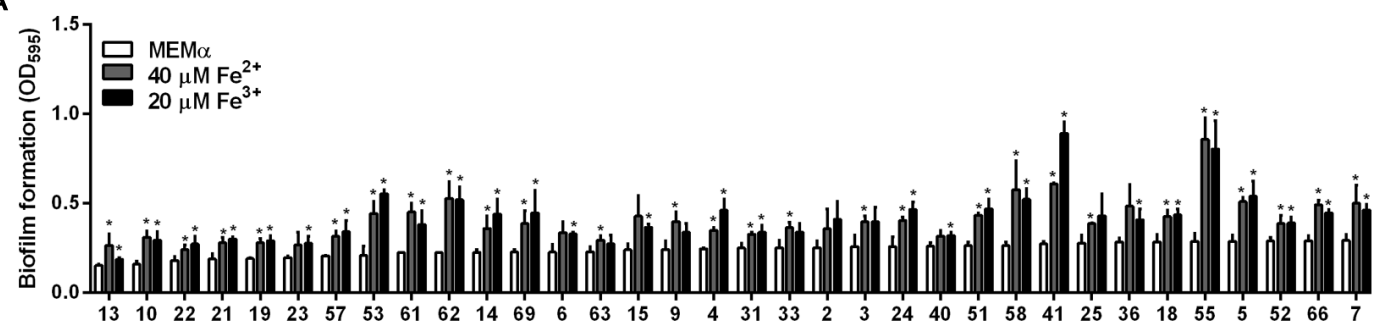

B

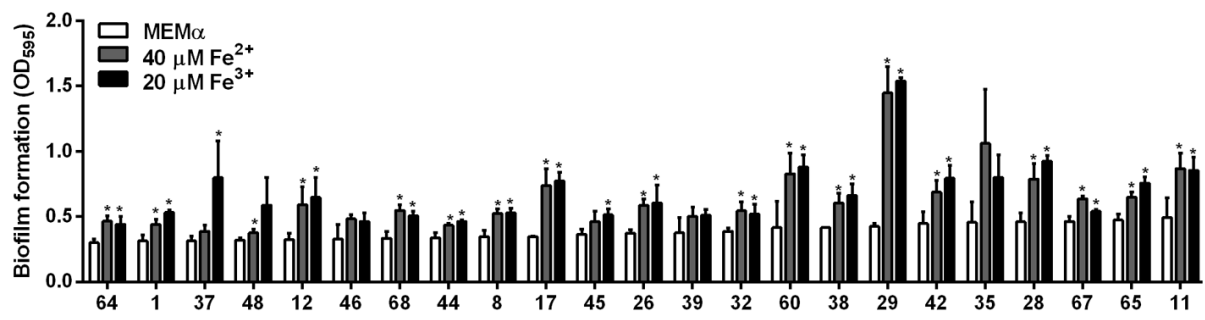

C

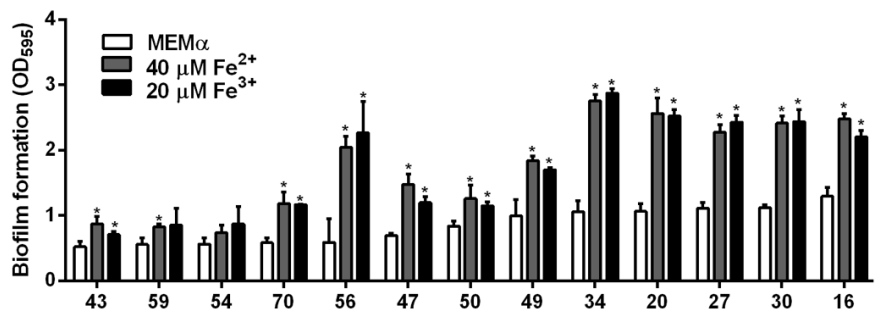

FIGURE 4 | Effects of iron (40 $\mu \mathrm{M} \mathrm{Fe}^{2+}$ and $20 \mu \mathrm{M} \mathrm{Fe}^{3+}$ ) on biofilm formation in 70 chicken isolates of $C$. jejuni that inherently form biofilms at low (A), medium (B), and high (C) levels. The inherent capability of biofilm formation was determined based on the $\mathrm{OD}_{595}$ in the biofilm assay. The low-, medium-, and high-level biofilm formers were those that generated $\mathrm{OD}_{595}$ of less than 0.3 , between 0.3 and 0.5 , and greater than 0.5 , respectively, in the absence of iron. The results show the means and standard deviations of three samples in a single experiment. The experiments were repeated three times, and similar results were observed in all repeated experiments. The statistical analysis was performed with Student's $t$-test compared to the control without iron treatment. ${ }^{*} P<0.05$.

C. jejuni. Genes encoding iron transporters, which are found in the genome of some $C$. jejuni strains, have been found to be non-functional or absent in other strains (Miller et al., 2009). Presumably, the diversities in the genes involved in iron uptake might be associated with the strain-dependent variations in iron concentrations affecting biofilm stimulation. We could not observe any correlation between the MLST CCs and the levels of biofilm formation (Figure 5B), presumably because the MLST scheme is based on the polymorphisms of the seven housekeeping genes ( $\operatorname{sp} p A, g \ln A, g l t A, g l y A, p g m$, tkt, and uncA) (Dingle et al., 2001). However, biofilm formation is complicated and involves proteins of various biological processes, such as motility, chemotaxis, oxidative stress response, heat shock response, and energy generation (Kalmokoff et al., 2006). This might be the reason why the MLST sequence types were not well correlated with biofilm formation in this study.

Biofilm formation in $P$. aeruginosa is inhibited by lactoferrin, a mammalian iron chelator (Singh et al., 2002). The sequestration of iron by lactoferrin reduces the intracellular levels of iron and inhibits biofilm formation (Banin et al., 2005). The antibiofilm effect of lactoferrin in $P$. aeruginosa involves incessant twitching motility that affects bacterial attachment to a surface and microcolony formation during biofilm development (Singh et al., 2002). Twitching motility is mediated by type IV pili (Mattick, 2002). Similarly, iron stimulates biofilm formation in E. coli by controlling the expression of type I fimbriae (Wu and Outten, 2009). However, C. jejuni does not produce pili (Gaynor et al., 2001). This indicates that a different mechanism may be involved in iron-mediated biofilm promotion in C. jejuni.

It has been reported that oxidative stress affects biofilm formation in C. jejuni (Kim et al., 2015). A mutation of $a h p C$ leads to the accumulation of total ROS and lipid hydroperoxides and enhances biofilm formation in C. jejuni, and antioxidant treatment inhibits the biofilm promotion by an $a h p C$ mutation (Oh and Jeon, 2014). PerR and CosR, key oxidative stress defense regulators in C. jejuni, also consistently affect biofilm formation in C. jejuni (Oh and Jeon, 2014; Turonova et al., 2015). Although C. jejuni is microaerophilic, interestingly, aerobic exposure facilitates biofilm formation in C. jejuni (Reuter et al., 2010). Our previous study has shown that oxidative stress plays a role in biofilm stimulation in C. jejuni under aerobic conditions (Oh et al., 2016). Oxidative 
A

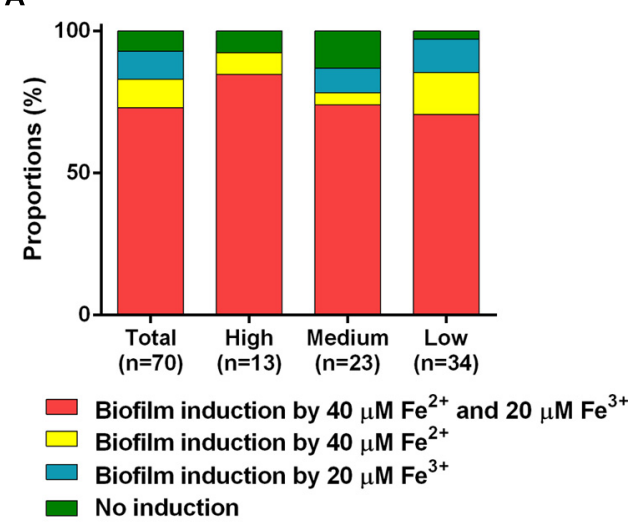

B

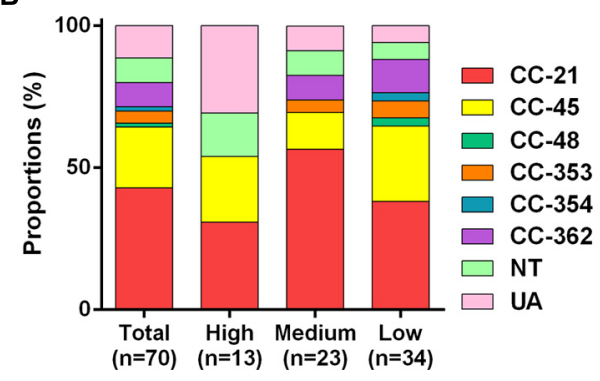

FIGURE 5 | MLST clonal complexes (CCs) of 70 tested strains of $C$. jejuni with different biofilm formation capabilities (A), and the proportions of the C. jejuni strains whose biofilm formation was enhanced by $40 \mu \mathrm{M} \mathrm{Fe}^{2+}$ and/or $20 \mu \mathrm{M} \mathrm{Fe}^{3+}$ (B). The low-, medium-, and high-level biofilm formers are the strains that generated $\mathrm{OD}_{595}$ of less than 0.3 , between 0.3 and 0.5 , and greater than 0.5, respectively, in the absence of iron. The MLST CCs of the 70 C. jejuni strains tested in the study were determined in our previous report (Oh et al., 2015).

stress affects biofilm formation in some other bacteria. Biofilm formation in Mycobacterium avium increases by the autoinducer2 (AI-2) signaling molecules by the induction of oxidative stress response involving the upregulation of genes encoding alkyl hydroperoxidases (e.g., $a h p C$ and $a h p D$ ), not through quorum sensing (Geier et al., 2008). An ahpC mutation in Acinetobacter oleivorans DR1 increases the accumulation of $\mathrm{H}_{2} \mathrm{O}_{2}$ in the cell, which enhances biofilm formation by the induction of exopolysaccharide production in biofilms (Jang et al., 2016). In C. jejuni, iron supplementation increased the accumulation of total ROS (Figure 2B) and the production of eDNA and extracellular polysaccharides in C. jejuni biofilms (Figure 3). Aerobic exposure and iron supplementation commonly result in the increase in oxidative stress. Based on the findings of this study, biofilm stimulation by iron through oxidative stress in C. jejuni may involve EPS production (Figure 3). EPS constitutes over $90 \%$ of the dry mass of biofilms (Flemming and Wingender, 2010) and contributes to the nutrient acquisition and desiccation tolerance (Flemming et al., 2016). Similarly, biofilm stimulation by iron in $S$. aureus is mediated by the increased production of polysaccharide intercellular adhesin (i.e., $\beta$-1,6-linked $\mathrm{N}$-acetyl glucosamine polymer) involved in biofilm formation (Lin et al., 2012). Exposure to increased iron concentrations augments the accumulation of ROS in C. jejuni (Figure 2B) and the production of EPS in biofilms (Figure 3). Presumably, the enhanced production of EPS by iron may help C. jejuni to reduce exposure to oxygen and other stress conditions by facilitating the formation of biofilm matrices encasing C. jejuni.

Cationic metal ions can be toxic for planktonic bacterial cells at high concentrations; however, the absorption and accumulation of metal ions in biofilms stabilizes biofilms and prevents their erosion by shear forces in B. subtilis (Grumbein et al., 2014). EDTA disrupts $P$. aeruginosa biofilms and enhances the dispersal of bacterial cells from biofilms (Banin et al., 2006). However, $\mathrm{Mg}^{2+}, \mathrm{Ca}^{2+}$, and $\mathrm{Fe}^{2+}$ inhibit the effect of EDTA on biofilm disruption, suggesting that divalent cations are important components that stabilize biofilms in $P$. aeruginosa (Banin et al., 2006). In addition to the effect of iron on oxidative stress, we cannot exclude the possibility that iron may also be involved in the stabilization of biofilm structure in C. jejuni. Future studies are required to elucidate the molecular mechanisms underlying the interplay between iron and oxidative stress in biofilm formation in C. jejuni.

\section{MATERIALS AND METHODS}

\section{Bacterial Strains and Culture Conditions}

Campylobacter jejuni NCTC 11168, the first genome-sequenced strain, was primarily used in this study. Seventy strains of C. jejuni were isolated from raw chicken in our previous study (Oh et al., 2015). The C. jejuni strains were routinely maintained at $37^{\circ} \mathrm{C}$ under microaerobic conditions $\left(5 \% \mathrm{O}_{2}, 10 \% \mathrm{CO}_{2}\right.$, and $\left.85 \% \mathrm{~N}_{2}\right)$ on either Mueller-Hinton agar plates or MEM $\alpha$ (Gibco, \#41061029), which does not contain iron. The microaerobic conditions were generated using a cylinder containing the premixed gas.

\section{Biofilm Assays}

Biofilm assays were carried out according to a protocol described in our previous study using MEM $\alpha$ (Oh and Jeon, 2014). Briefly, bacterial suspension was prepared from an overnight culture and then diluted with fresh $\mathrm{MEM} \alpha$ to an $\mathrm{OD}_{600}$ of 0.07 and placed into a 96-well plate (Corning, \#3595) in the presence of iron $\left(\mathrm{Fe}^{2+}\right.$ or $\left.\mathrm{Fe}^{3+}\right)$, iron chelator (Deferoxamine mesylate, DFMS), or antioxidant (N-acetyl-L-cysteine, NAC). After $24 \mathrm{~h}$, biofilms were washed twice with PBS ( $\mathrm{pH} 7.4$ ) and stained with $1 \%$ crystal violet. The dye eluted with the elution buffer (10\% acetic acid and 30\% methanol) was measured with a plate reader (FLUOstar Omega; BMG Labtech, Germany) at $595 \mathrm{~nm}$. For bacterial counting of biofilms, C. jejuni biofilm samples were washed twice with PBS and resuspended in fresh $\mathrm{MH}$ broth. The resuspended biofilm samples were serially diluted in $\mathrm{MH}$ broth and spread on $\mathrm{MH}$ agar for enumeration. The experiment was conducted with triplicate samples and independently repeated at least three times. 
A

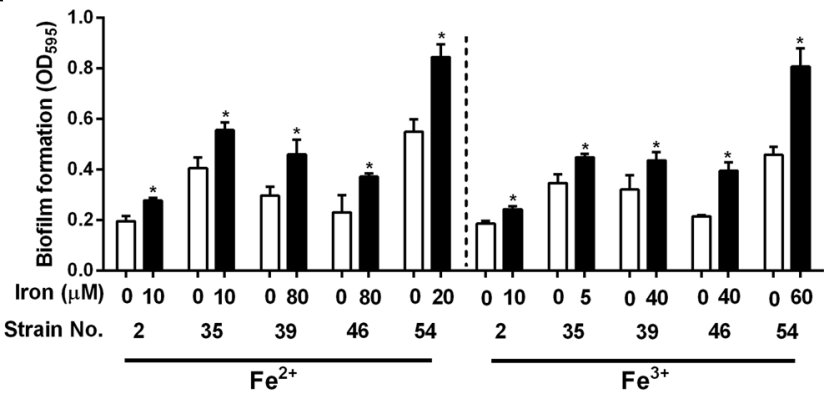

B

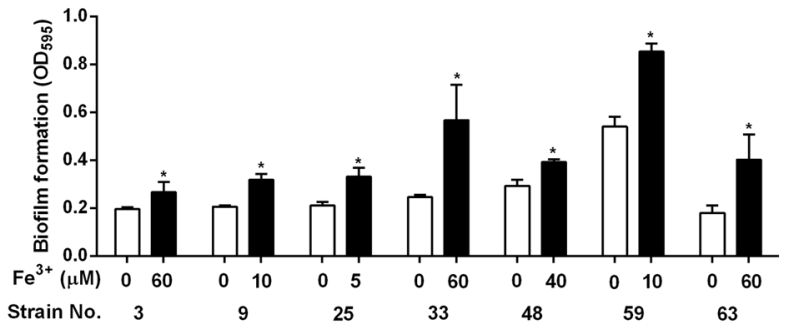

C

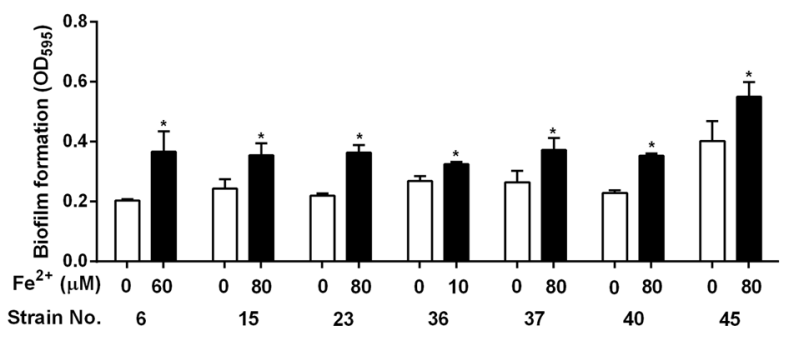

FIGURE 6 | Biofilm stimulation at different iron concentrations in the C. jejuni strains whose biofilm formation was enhanced by neither $40 \mu \mathrm{M}$ Fe $\mathrm{e}^{2+}$ nor $20 \mu \mathrm{M}$ Fe $\mathrm{F}^{3+}$ (A), by $40 \mu \mathrm{M} \mathrm{Fe}^{2+}$ but not by $20 \mu \mathrm{M} \mathrm{Fe}^{3+}$ (B), and by $20 \mu \mathrm{M} \mathrm{Fe}^{3+}$ but not by $40 \mu \mathrm{M} \mathrm{Fe}^{2+}$ (C). The results show the means and standard deviations of three samples in a single experiment. Similar results were observed in all experiments repeated three times independently. The statistical analysis was performed with Student's $t$-test in comparison with the control without iron treatment. ${ }^{*} P<0.05$.

\section{Measurement of Total ROS}

The total ROS level in biofilms was determined with CM$\mathrm{H}_{2}$ DCFDA (Thermo Fisher, United States), a general oxidative stress indicator, according to our previous study (Oh and Jeon, 2014). Briefly, biofilms were washed twice with PBS and re-suspended in PBS ( $\mathrm{pH}$ 7.4). After addition of $10 \mu \mathrm{M} \quad \mathrm{CM}-\mathrm{H}_{2} \mathrm{DCFDA}$, fluorescence was measured with a fluorometer (FLUOstar Omega) at ex $485 \mathrm{~nm} / \mathrm{em} 520 \mathrm{~nm}$. The total ROS levels were normalized with the total protein amounts that were determined using a Bradford assay. The experiment was conducted with triplicate samples and independently repeated three times.

\section{Fluorescence Microscopic Analysis of Biofilms}

Biofilm formation was also analyzed with fluorescence microscopy. Biofilms were developed on a circle cover glass in a 24-well plate for $24 \mathrm{~h}$ at $37^{\circ} \mathrm{C}$ under microaerobic conditions. Biofilm samples were washed twice with PBS and fixed with $4 \%$ paraformaldehyde for $30 \mathrm{~min}$ at room temperature. The biofilms were then washed with PBS and stained with SYTO9, BOBO3, and CW to detect total (both intracellular and extracellular) DNA, intracellular DNA, and extracellular polysaccharides, respectively. CW binds $\beta 1-3$ and $\beta 1-4$ carbohydrate linkages and was previously used to detect extracellular polysaccharides in biofilms (McLennan et al., 2008). After washing, the biofilms were analyzed with a fluorescence microscope (Carl Zeiss, Axio Imager A1). The experiment was repeated three times.

\section{Measurement of eDNA in Biofilms}

The isolation of eDNA from biofilms was performed as described previously ( $\mathrm{Wu}$ and $\mathrm{Xi}, 2009$ ). After washing twice with PBS, biofilms were harvested with $2 \%$ EDTA and incubated at $4^{\circ} \mathrm{C}$ for $3 \mathrm{~h}$ with shaking (250 rpm). An equal volume of $2 \%$ cetyltrimethyl ammonium bromide (CTAB) was added, and the suspension was incubated on ice for $1 \mathrm{~h}$. After centrifugation at $10,000 \times g$ for $10 \mathrm{~min}$, the pellet was re-suspended in TE buffer, and an 
equal volume of phenol: chloroform: isoamyl alcohol (25: 24: 1) solution was added. After centrifugation, the top phase of each sample was transferred to a new tube, and $2 \times$ volume of icecold ethanol and $1 / 10 \times$ volume of $3 \mathrm{M}$ sodium acetate were added. After incubation at $-20^{\circ} \mathrm{C}$ for $1 \mathrm{~h}$, the pellets were washed twice with $70 \%$ ethanol. After dissolving with water, DNA concentrations were measured with a spectrophotometer and normalized with the amount of total proteins in biofilms that was determined with a Bradford assay. The experiment was conducted with triplicate samples and repeated three times.

\section{Measurement of Intracellular Iron Levels}

The intracellular iron concentration was measured as described previously with slight modifications (Riemer et al., 2004). Briefly, biofilms were washed twice with PBS and disrupted with a sonicator (BioRuptor Plus; Diagenode, United States). The biofilm samples were mixed with an iron-detection reagent (6.5 mM ferrozine, $6.5 \mathrm{mM}$ neocuproine, $2.5 \mathrm{M}$ ammonium acetate, and $1 \mathrm{M}$ ascorbic acid) and incubated at room temperature for $30 \mathrm{~min}$. The absorbance at $550 \mathrm{~nm}$ was measured with a plate reader (FLUOstar Omega). Intracellular iron levels were normalized with protein concentrations that were determined with a Bradford assay. The experiment was conducted with triplicate samples and independently repeated three times.

\section{REFERENCES}

Al-Haideri, H., White, M. A., and Kelly, D. J. (2016). Major contribution of the type II beta carbonic anhydrase CanB (Cj0237) to the capnophilic growth phenotype of Campylobacter jejuni. Environ. Microbiol. 18, 721-735. doi: 10.1111/14622920.13092

Asakura, H. I, Yamasaki, M., Yamamoto, S., and Igimi, S. (2007). Deletion of peb4 gene impairs cell adhesion and biofilm formation in Campylobacter jejuni. FEMS Microbiol. Lett. 275, 278-285. doi: 10.1111/j.1574-6968.2007.00893.x

Banin, E., Brady, K. M., and Greenberg, E. P. (2006). Chelator-induced dispersal and killing of Pseudomonas aeruginosa cells in a biofilm. Appl. Environ. Microbiol. 72, 2064-2069. doi: 10.1128/AEM.72.3.2064-2069.2006

Banin, E., Vasil, M. L., and Greenberg, E. P. (2005). Iron and Pseudomonas aeruginosa biofilm formation. Proc. Natl. Acad. Sci. U.S.A. 102, 11076-11081. doi: 10.1073/pnas.0504266102

Berlutti, F., Ajello, M., Bosso, P., Morea, C., Petrucca, A., Antonini, G., et al. (2004). Both lactoferrin and iron influence aggregation and biofilm formation in Streptococcus mutans. Biometals 17, 271-278. doi: 10.1023/B: BIOM.0000027704.53859.d3

Branda, S. S., Vik, S., Friedman, L., and Kolter, R. (2005). Biofilms: the matrix revisited. Trends Microbiol. 13, 20-26. doi: 10.1016/j.tim.2004.11.006

Buswell, C. M., Herlihy, Y. M., Lawrence, L. M., Mcguiggan, J. T., Marsh, P. D., Keevil, C. W., et al. (1998). Extended survival and persistence of Campylobacter spp. in water and aquatic biofilms and their detection by immunofluorescent-antibody and -rRNA staining. Appl. Environ. Microbiol. 64, 733-741.

Chandrangsu, P., Rensing, C., and Helmann, J. D. (2017). Metal homeostasis and resistance in bacteria. Nat. Rev. Microbiol. 15, 338-350. doi: 10.1038/nrmicro. 2017.15

Cornelis, P., Wei, Q., Andrews, S. C., and Vinckx, T. (2011). Iron homeostasis and management of oxidative stress response in bacteria. Metallomics 3, 540-549. doi: $10.1039 / \mathrm{clmt} 00022 \mathrm{e}$

Davis, L., and DiRita, V. (2008). Growth and laboratory maintenance of Campylobacter jejuni. Curr. Protoc. Microbiol. Chapter 8:Unit 8A.1.1-8A.1.7. doi: 10.1002/9780471729259.mc08a01s10

\section{Statistical Analysis}

The statistical analysis was performed with Student's $t$-test in comparison with the control without iron treatment using GraphPad Prism 6 (GraphPad Software, La Jolla, CA, United States).

\section{AUTHOR CONTRIBUTIONS}

$\mathrm{EO}$ and $\mathrm{BJ}$ designed the project. $\mathrm{EO}$ and $\mathrm{KA}$ performed the experiments. $\mathrm{EO}$ and $\mathrm{BJ}$ data analysis. $\mathrm{EO}, \mathrm{KA}$, and $\mathrm{BJ}$ wrote the manuscript.

\section{FUNDING}

This study was supported by NSERC Discovery Grant (4018432012-RGPIN) and the Canada Foundation for Innovation (CFI) to BJ.

\section{SUPPLEMENTARY MATERIAL}

The Supplementary Material for this article can be found online at: https://www.frontiersin.org/articles/10.3389/fmicb. 2018.01204/full\#supplementary-material

Dingle, K., Colles, F., Wareing, D., Ure, R., Fox, A., Bolton, F., et al. (2001). Multilocus sequence typing system for Campylobacter jejuni. J. Clin. Microbiol. 39, 14-23. doi: 10.1128/JCM.39.1.14-23.2001

Flemming, H. C., and Wingender, J. (2010). The biofilm matrix. Nat. Rev. Microbiol. 8, 623-633. doi: 10.1038/nrmicro2415

Flemming, H. C., Wingender, J., Szewzyk, U., Steinberg, P., Rice, S. A., and Kjelleberg, S. (2016). Biofilms: an emergent form of bacterial life. Nat. Rev. Microbiol. 14, 563-575. doi: 10.1038/nrmicro.2016.94

Gaynor, E. C., Ghori, N., and Falkow, S. (2001). Bile-induced 'pili' in Campylobacter jejuni are bacteria-independent artifacts of the culture medium. Mol. Microbiol. 39, 1546-1549. doi: 10.1046/j.1365-2958.2001.02341.x

Geier, H., Mostowy, S., Cangelosi, G. A., Behr, M. A., and Ford, T. E. (2008). Autoinducer-2 triggers the oxidative stress response in Mycobacterium avium, leading to biofilm formation. Appl. Environ. Microbiol. 74, 1798-1804. doi: 10.1128/AEM.02066-07

Grumbein, S., Opitz, M., and Lieleg, O. (2014). Selected metal ions protect Bacillus subtilis biofilms from erosion. Metallomics 6, 1441-1450. doi: 10.1039/ c4mt00049h

Hermans, D., Pasmans, F., Messens, W., Martel, A., Van Immerseel, F., Rasschaert, G., et al. (2012). Poultry as a host for the zoonotic pathogen Campylobacter jejuni. Vector Borne Zoonotic Dis. 12, 89-98. doi: 10.1089/vbz. 2011.0676

Huang, H., Brooks, B. W., Lowman, R., and Carrillo, C. D. (2015). Campylobacter species in animal, food, and environmental sources, and relevant testing programs in Canada. Can. J. Microbiol. 61, 701-721. doi: 10.1139/cjm-20140770

Jang, I. A., Kim, J., and Park, W. (2016). Endogenous hydrogen peroxide increases biofilm formation by inducing exopolysaccharide production in Acinetobacter oleivorans DR1. Sci. Rep. 6:21121. doi: 10.1038/srep21121

Johnson, M., Cockayne, A., Williams, P. H., and Morrissey, J. A. (2005). Ironresponsive regulation of biofilm formation in Staphylococcus aureus involves fur-dependent and fur-independent mechanisms. J. Bacteriol. 187, 8211-8215. doi: 10.1128/JB.187.23.8211-8215.2005

Jokinen, C., Edge, T. A., Ho, S., Koning, W., Laing, C., Mauro, W., et al. (2011). Molecular subtypes of Campylobacter spp., Salmonella enterica, and Escherichia 
coli O157:H7 isolated from faecal and surface water samples in the Oldman River watershed, Alberta, Canada. Water Res. 45, 1247-1257. doi: 10.1016/j. watres.2010.10.001

Kalmokoff, M., Lanthier, P., Tremblay, T.-L., Foss, M., Lau, P. C., Sanders, G., et al. (2006). Proteomic analysis of Campylobacter jejuni 11168 biofilms reveals a role for the motility complex in biofilm formation. J. Bacteriol. 188, 4312-4320. doi: 10.1128/JB.01975-05

Kemp, R., Leatherbarrow, A., Williams, N., Hart, C., Clough, H., Turner, J., et al. (2005). Prevalence and genetic diversity of Campylobacter spp. in environmental water samples from a 100-square-kilometer predominantly dairy farming area. Appl. Environ. Microbiol. 71, 1876-1882. doi: 10.1128/AEM. 71.4.1876-1882.2005

Kim, J.-C., Oh, E., Kim, J., and Jeon, B. (2015). Regulation of oxidative stress resistance in Campylobacter jejuni, a microaerophilic foodborne pathogen. Front. Microbiol. 6:751. doi: 10.3389/fmicb.2015.00751

Kirk, M. D., Pires, S. M., Black, R. E., Caipo, M., Crump, J. A., Devleesschauwer, B., et al. (2015). World Health Organization estimates of the global and regional disease burden of 22 foodborne bacterial, protozoal, and viral diseases, 2010: a data synthesis. PLoS Med. 12:e1001921. doi: 10.1371/journal.pmed.1001921

Lin, M. H., Shu, J. C., Huang, H. Y., and Cheng, Y. C. (2012). Involvement of iron in biofilm formation by Staphylococcus aureus. PLoS One 7:e34388. doi: 10.1371/journal.pone.0034388

Maal-Bared, R., Bartlett, K. H., Bowie, W. R., and Hall, E. R. (2012). Campylobacter spp. distribution in biofilms on different surfaces in an agricultural watershed (Elk Creek, British Columbia): using biofilms to monitor for Campylobacter. Int. J. Hyg. Environ. Health 215, 270-278. doi: 10.1016/j.ijheh.2011.12.004

Mattick, J. S. (2002). Type IV pili and twitching motility. Annu. Rev. Microbiol. 56, 289-314. doi: 10.1146/annurev.micro.56.012302.160938

McLennan, M. K., Ringoir, D. D., Frirdich, E., Svensson, S. L., Wells, D. H., Jarrell, H., et al. (2008). Campylobacter jejuni biofilms up-regulated in the absence of the stringent response utilize a calcofluor white-reactive polysaccharide. J. Bacteriol. 190, 1097-1107. doi: 10.1128/JB.00516-07

Miller, C. E., Williams, P. H., and Ketley, J. M. (2009). Pumping iron: mechanisms for iron uptake by Campylobacter. Microbiology 155, 3157-3165. doi: 10.1099/ mic.0.032425-0

Murphy, C., Carroll, C., and Jordan, K. N. (2006). Environmental survival mechanisms of the foodborne pathogen Campylobacter jejuni. J. Appl. Microbiol. 100, 623-632. doi: 10.1111/j.1365-2672.2006.02903.x

Naikare, H., Palyada, K., Panciera, R., Marlow, D., and Stintzi, A. (2006). Major role for FeoB in Campylobacter jejuni ferrous iron acquisition, gut colonization, and intracellular survival. Infect. Immun. 74, 5433-5444. doi: 10.1128/IAI.00052-06

Oh, E., and Jeon, B. (2014). Role of alkyl hydroperoxide reductase (AhpC) in the biofilm formation of Campylobacter jejuni. PLoS One 9:e87312. doi: 10.1371/ journal.pone.0087312

Oh, E., Kim, J. C., and Jeon, B. (2016). Stimulation of biofilm formation by oxidative stress in Campylobacter jejuni under aerobic conditions. Virulence 7, 846-851. doi: 10.1080/21505594.2016.1197471

Oh, E., Mcmullen, L., and Jeon, B. (2015). High prevalence of hyper-aerotolerant Campylobacter jejuni in retail poultry with potential implication in human infection. Front. Microbiol. 6:1263. doi: 10.3389/fmicb.2015.01263
Palyada, K., Threadgill, D., and Stintzi, A. (2004). Iron acquisition and regulation in Campylobacter jejuni. J. Bacteriol. 186, 4714-4729. doi: 10.1128/JB.186.14. 4714-4729.2004

Reeser, R. J., Medler, R. T., Billington, S. J., Jost, B. H., and Joens, L. A. (2007). Characterization of Campylobacter jejuni biofilms under defined growth conditions. Appl. Environ. Microbiol. 73, 1908-1913. doi: 10.1128/AEM. 00740-06

Reuter, M., Mallett, A., Pearson, B. M., and Van Vliet, A. H. (2010). Biofilm formation by Campylobacter jejuni is increased under aerobic conditions. Appl. Environ. Microbiol. 76, 2122-2128. doi: 10.1128/AEM.01878-09

Riemer, J., Hoepken, H. H., Czerwinska, H., Robinson, S. R., and Dringen, R. (2004). Colorimetric ferrozine-based assay for the quantitation of iron in cultured cells. Anal. Biochem. 331, 370-375. doi: 10.1016/j.ab.2004. 03.049

Silva, J., Leite, D., Fernandes, M., Mena, C., Gibbs, P. A., and Teixeira, P. (2011). Campylobacter spp. as a foodborne pathogen: a review. Front. Microbiol. 2:200. doi: $10.3389 /$ fmicb.2011.00200

Singh, P. K., Parsek, M. R., Greenberg, E. P., and Welsh, M. J. (2002). A component of innate immunity prevents bacterial biofilm development. Nature 417, 552-555. doi: 10.1038/417552a

Sulaeman, S., Hernould, M., Schaumann, A., Coquet, L., Bolla, J. M., Dé, E., et al. (2012). Enhanced adhesion of Campylobacter jejuni to abiotic surfaces is mediated by membrane proteins in oxygen-enriched conditions. PLoS One 7:e46402. doi: 10.1371/journal.pone.0046402

Turonova, H., Briandet, R., Rodrigues, R., Hernould, M., Hayek, N., Stintzi, A., et al. (2015). Biofilm spatial organization by the emerging pathogen Campylobacter jejuni: comparison between NCTC 11168 and 81-176 strains under microaerobic and oxygen-enriched conditions. Front. Microbiol. 6:709. doi: $10.3389 /$ fmicb.2015.00709

Willison, H. J., Jacobs, B. C., and Van Doorn, P. A. (2016). Guillain-Barre syndrome. Lancet 388, 717-727. doi: 10.1016/S0140-6736(16)00339- 1

$\mathrm{Wu}, \mathrm{J}$., and $\mathrm{Xi}, \mathrm{C}$. (2009). Evaluation of different methods for extracting extracellular DNA from the biofilm matrix. Appl. Environ. Microbiol. 75, 5390-5395. doi: 10.1128/AEM.00400-09

Wu, Y., and Outten, F. W. (2009). IscR controls iron-dependent biofilm formation in Escherichia coli by regulating type I fimbria expression. J. Bacteriol. 191, 1248-1257. doi: 10.1128/JB.01086-08

Conflict of Interest Statement: The authors declare that the research was conducted in the absence of any commercial or financial relationships that could be construed as a potential conflict of interest.

The reviewer GP and handling Editor declared their shared affiliation.

Copyright (c) $2018 \mathrm{Oh}$, Andrews and Jeon. This is an open-access article distributed under the terms of the Creative Commons Attribution License (CC BY). The use, distribution or reproduction in other forums is permitted, provided the original author(s) and the copyright owner are credited and that the original publication in this journal is cited, in accordance with accepted academic practice. No use, distribution or reproduction is permitted which does not comply with these terms. 